Audiology

Neurotology
Audiol Neurotol 2013;18:71-82

DOI: $\underline{10.1159 / 000343909}$
Received: June 16, 2012

Accepted after revision: October 1, 2012

Published online: November 6, 2012

\title{
Time-Frequency Analysis of Transient-Evoked Otoacoustic Emissions in Children Exposed to Carboplatin Chemotherapy
}

\author{
Shaum Bhagat ${ }^{a, b}$ Johnnie Bass ${ }^{b}$ Ibrahim Qaddoumi ${ }^{b}$ Rachel Brennan ${ }^{b}$ \\ Matthew Wilson ${ }^{b, c}$ Jianrong Wu ${ }^{\text {b }}$ Carlos-Rodriguez Galindo b, d \\ Alessia Paglialonga ${ }^{\mathrm{e}}$ Gabriella Tognola ${ }^{\mathrm{e}}$ \\ ${ }^{a}$ Hearing Science Laboratory, School of Communication Sciences and Disorders, The University of Memphis, \\ ${ }^{b}$ St. Jude Children's Research Hospital, and 'The University of Tennessee Health Science Center, Memphis, Tenn., \\ and ${ }^{d}$ Dana-Farber Cancer Institute, Harvard Medical School, Boston, Mass., USA; ${ }^{e}$ Istituto di Ingegneria Biomedica, \\ Consiglio Nazionale delle Ricerche, Milano, Italy
}

\section{Key Words}

Retinoblastoma $\cdot$ Carboplatin therapy $\cdot$ Hearing loss

Ototoxicity $\cdot$ Transient-evoked otoacoustic emissions

\section{Abstract}

The aims of this study were to characterize and quantify time-frequency changes in transient-evoked otoacoustic emissions (TEOAEs) recorded in children diagnosed with retinoblastoma who were receiving carboplatin chemotherapy. A signal processing technique, the wavelet transform (WT), was used to analyze TEOAE waveforms in narrow-band frequency components. Ten children (aged 3-72 months) diagnosed with unilateral or bilateral retinoblastoma were enrolled in the study. TEOAEs were acquired from the children with linear sequences of $70 \mathrm{~dB}$ peak equivalent SPL clicks. After WT analysis, TEOAE energy, latency and normalized energy in the narrow-band frequency components were compared before and during carboplatin chemotherapy treatment (average dose $1693 \mathrm{mg} / \mathrm{m}^{2}$ ). On a group basis, no significant differences $(p>0.05)$ in the TEOAE energy, latency or normalized energy before and after carboplatin treatment were observed. There were decreases in normalized energy on an individual basis in 10 out of 18 ears in the sam- ple. Exposure to carboplatin chemotherapy did not cause significant changes in TEOAE energy, latency and normalized energy during treatment. However, long-term monitoring of hearing with measurements of TEOAEs is warranted, given the risks of delayed hearing loss in some children receiving carboplatin chemotherapy.

Copyright $\odot 2012$ S. Karger AG, Basel

\section{Introduction}

Carboplatin (cis-diammine [1,1- cyclobutanedicarboxylate]-platinum [II]) is a second-generation platinum compound that was initially reported to have less ototoxic side effects than cisplatin in children [Bacha et al., 1986]. More recently, clinical studies of carboplatin ototoxicity conducted in children have revealed conflicting results. Macdonald et al. [1994] found that $50 \%$ of the children in their study had a sensorineural hearing loss

S.B. and J.B. contributed equally to this paper. Portions of the paper were presented at AudiologyNow! in Boston, Mass., on March 30, 2012.

\section{KARGER \\ Fax +4161306 1234 \\ E-Mail karger@karger.ch}

www.karger.com
(C) 2012 S. Karger AG, Basel

$1420-3030 / 13 / 0182-0071 \$ 38.00 / 0$

Accessible online at:

www.karger.com/aud
Shaum P. Bhagat

Hearing Science Laboratory, School of Communication Sciences and Disorder The University of Memphis, 807 Jefferson Avenue

Memphis, TN 38105 (USA)

E-Mail sbhagat@memphis.edu 
in the 4000-12000 $\mathrm{Hz}$ range following treatment with carboplatin. They found that hearing losses could occur after the first dose of carboplatin and could progress with subsequent doses. Similarly, Simon et al. [2002] reported that $40 \%$ of the children treated with high-dose carboplatin developed a hearing impairment and Knight et al. [2005] found that $38 \%$ of the children treated with carboplatin developed sensorineural hearing loss. In contrast, other studies have found that ototoxic complications from carboplatin chemotherapy were rare and mild in severity [Stern and Bunin, 2002; Bertolini et al., 2004; Dean et al., 2008]. The variability of carboplatin ototoxicity seen across past studies may be related to insufficient control of confounding factors. Factors that may potentiate the severity of carboplatin ototoxicity include prior exposure to cisplatin or other ototoxic medications and high dosage of carboplatin associated with autologous stem cell reinfusion [Parsons et al., 1998; Knight et al., 2005]. Another factor that may increase the severity of platinum-compound ototoxicity is patient age, with younger children being more susceptible than older children [Li et al., 2004; Qaddoumi et al., 2012].

Children diagnosed with retinoblastoma are among the youngest-identified cancer victims. Retinoblastoma is an uncommon malignant intraocular tumor with a mean age-adjusted incidence of 11.8 per million children [Broaddus et al., 2009]. The vast majority of children with retinoblastoma are diagnosed before the age of 3 years, with cases of bilateral retinoblastoma usually recognized at an earlier age than cases of unilateral retinoblastoma [Abramson et al., 1998; Broaddus et al., 2009]. Traditionally, the treatment options for retinoblastoma include enucleation (removal of the affected eye or eyes) and external beam radiation therapy. Due to efforts to preserve vision and avoid facial disfigurements from radiation, a more conservative approach with systematic chemotherapy is often recommended in children meeting the criteria for this form of treatment. Carboplatin is often included in multi-drug chemotherapy regimens designed to treat retinoblastoma [Rodriguez-Galindo et al., 2003]. The pathophysiology of carboplatin ototoxicity is not completely understood, but evidence from animal models suggests dose-dependent and species-specific effects. In chinchillas, low doses of carboplatin cause progressive inner hair cell (IHC) and spiral ganglion neuron loss from the apex to the base of the cochlea, and outer hair cells (OHCs) are largely unaffected [Takeno et al., 1994; Hofstetter et al., 1997]. At higher doses of carboplatin, extensive loss of IHCs is exhibited across all cochlear turns and loss of OHCs is exhibited most prominently in the basal turn [Hofstetter et al., 1997; Bauer and Brozowski, 2005]. Studies of high-dose carboplatin administration in guinea pigs reveal that primarily $\mathrm{OHCs}$ are destroyed [Saito et al., 1989].

Given the potential for damage to cochlear structures, monitoring for carboplatin ototoxicity in pediatric cancer patients is a priority. Early detection of potential ototoxicity from carboplatin can lead to clinical decisions to lower drug dosage to preserve residual hearing or can lead to enrollment in early intervention programs in cases where the dosage cannot be modified. In addition, children with retinoblastoma have visual impairment in one or both eyes, making it crucial to recognize if carboplatin is potentially causing an additional sensory handicap.

Behavioral hearing tests can be difficult to conduct or interpret in young children or infants. Studies have shown that otoacoustic emission (OAE) tests are an effective alternative to behavioral hearing assessments in monitoring cochlear function in children exposed to ototoxic drugs [Stavroulaki et al., 2001; Knight et al., 2007]. OAEs are usually inaudible sounds that can be measured in an ear canal by a probe assembly containing a sensitive microphone. It is believed that OAEs originate from $\mathrm{OHC}$ oscillations that are requisite for the high sensitivity and sharp tuning of the healthy cochlea [Brownell, 1990]. Transient-evoked otoacoustic emissions (TEOAEs) are observed after acoustic stimulation of an ear with brief sounds such as clicks or tone bursts. As a noninvasive and indirect test of $\mathrm{OHC}$ function, measurements of TEOAEs have been informative in providing early evidence of $\mathrm{OHC}$ dysfunction in some individuals receiving platinum-compound chemotherapy. Yilmaz et al. [2010] found that in patients receiving cisplatin for lung cancer, changes in TEOAEs were detected before changes in pure-tone thresholds occurred. However, other studies have shown that TEOAEs are less sensitive than puretone thresholds at indicating the early onset of cisplatin ototoxicity [Allen et al., 1998; Yardley et al., 1998; Stavroulaki et al., 2001]. Clinical studies of carboplatin ototoxicity using TEOAEs are less prevalent in the literature. Yardley et al. [1998] found changes in TEOAEs in adult patients exposed to cisplatin, but no changes were seen in adult patients exposed to carboplatin. Since pure-tone thresholds are often difficult to measure in the youngest patients with retinoblastoma, it is imperative that an OAE test sensitive to the early effects of ototoxic drugs be utilized. Most conventional TEOAE tests utilize a Fast Fourier Transform (FFT) to analyze the TEOAE waveform across broadband or half-octave band regions. 
These FFT spectral components are measured before and after chemotherapy treatment to examine if a change in the level (in $\mathrm{dB}$ ) of a spectral component is reduced following exposure to the platinum compound. FFT analysis is best suited for stationary waveforms that do not exhibit changes in frequency over time. The TEOAE waveform is comprised of a summation of evoked responses from divergent cochlear locations, with each location tuned to a characteristic frequency. The latency of the response from each cochlear location contributing to the overall response is different from the base to the apex of the cochlea, producing a time-varying TEOAE waveform. FFT analysis may not capture the more subtle changes in the TEOAE waveform which are direct consequences of the time-varying behavior of the TEOAE signal that may signify the early onset of ototoxicity. A signal processing technique that is theoretically more suited to analyze with high accuracy the time-varying TEOAE waveform is the wavelet transform (WT) [Tognola et al., 1998]. Application of WT analysis allows the whole TEOAE waveform to be decomposed into a larger set of frequency components, each representing cochlear functioning within a narrow-band region along the basilar membrane. From both theoretical and practical points of view, a filter-bank approach based on the traditional FFT gives no information on how the frequency content of TEOAEs changes with time. As such, the traditional FFT analysis performs very well in clinical applications (such as in neonatal hearing screening) where it is not necessary to extract and analyze subtle features of the signal that are direct consequences of the time-varying behavior of TEOAEs. On the contrary, FFT analysis may not be accurate in all the other cases where it is necessary to extract parameters that may reveal subtle and subclinical alterations of the signal. Past studies [Tognola et al., 1997, 1998; Yang et al., 2002; Marozas et al., 2006; Zhang et al., 2008] demonstrated that in these latter cases, the most suitable approaches for the analysis of TEOAEs are those based on the WT, because of the sharp time and frequency resolutions these approaches have. The rationale behind the better performance of WT-based approaches in detecting subtle alterations in time-varying signals is that the time and frequency resolution of these methods is not fixed over the entire time-frequency plane but can vary. In this way, high-frequency components can be analyzed with a good time resolution while low-frequency components can be analyzed with a good frequency resolution. In particular, the WT analysis gives very accurate results if the signal is made up by low-frequency components of long duration and high-frequency components of brief

Time-Frequency Analysis of TEOAEs in Children on Carboplatin Chemotherapy duration, such as in the case of TEOAEs. Several studies have shown the usefulness of WT analysis when highly sensitive methods are needed to detect possible subtle changes in TEOAEs. Information gained from WT analysis of TEOAEs has led to insights concerning the development of cochlear mechanisms in newborns and young infants [Tognola et al., 2001; Moleti et al., 2005], the altered cochlear mechanisms underlying noise-induced hearing loss and tinnitus in adults [Tognola et al., 1999; Paglialonga et al., 2011b], the effects of exposure to electromagnetic fields on cochlear mechanisms in adults [Paglialonga et al., 2007], and the effects of deletion of the elastin gene on active cochlear mechanisms in patients with Williams syndrome [Paglialonga et al., 2011a].

To date, no study has utilized the WT analysis of TEOAEs in children exposed to platinum compounds. The aims of this study were to characterize and quantify time-frequency changes in TEOAEs recorded in children diagnosed with retinoblastoma who were receiving carboplatin chemotherapy. The children examined were evaluated in a protocol that utilized distortion-product otoacoustic emission (DPOAE) and TEOAE measurements. The DPOAE test results were reported in an earlier paper [Bhagat et al., 2010] and this paper presents the results from the TEOAE tests.

\section{Materials and Methods}

Subjects

The subjects were children undergoing treatment for retinoblastoma at St. Jude Children's Research Hospital as part of a frontline protocol. The study sample comprised 10 children $(5 \mathrm{fe}-$ males and 5 males). There were 5 cases of unilateral retinoblastoma and 5 of bilateral retinoblastoma. Age (rounded to the nearest month) at the onset of treatment ranged from 3 to 72 months. Demographic characteristics are listed in table 1 . This study was approved by the institutional review boards of the respective institutions of the researchers and informed consent was obtained from the childrens' parents/legal guardians.

\section{Drug Dosage Schedule}

Carboplatin was delivered intravenously (i.v.) at 3- to 4-week intervals. The children received 3-4 courses of chemotherapy. The dose of carboplatin received by each child at the midpoint of the chemotherapy regimen ranged from $1236-2210 \mathrm{mg} / \mathrm{m}^{2}$ (see table 1). The children also received vincristine and topotecan or etoposide either concurrently with or prior to the carboplatin treatments. Although the children received multiple chemotherapy drugs, only carboplatin has well-documented ototoxic side effects. The main side effect of vincristine is neurotoxicity, and it is not thought to damage OHCs or alter measurements of TEOAEs in adult human patients [Riga et al., 2006]. Similarly, exposure to topotecan did not affect measurements of TEOAEs in 
Table 1. Subject characteristics

\begin{tabular}{lclll}
\hline $\begin{array}{l}\text { Subject } \\
\text { No. }\end{array}$ & $\begin{array}{l}\text { Age at onset } \\
\text { of treatment } \\
\text { months }\end{array}$ & Gender & $\begin{array}{l}\text { Type of } \\
\text { retino- } \\
\text { blastoma }\end{array}$ & $\begin{array}{l}\text { Dose of } \\
\text { carboplatin } \\
\mathrm{mg} / \mathrm{m}^{2}\end{array}$ \\
\hline 002 & 8 & Male & unilateral & 1888 \\
003 & 9 & Female & unilateral & 2210 \\
004 & 10 & Female & bilateral & 2004 \\
005 & 3 & Male & bilateral & 1606 \\
006 & 5 & Female & bilateral & 1290 \\
007 & 72 & Male & unilateral & 1851 \\
010 & 4 & Female & unilateral & 1598 \\
012 & 10 & Female & bilateral & 1477 \\
014 & 11 & Male & bilateral & 1236 \\
016 & 7 & Male & unilateral & 1768 \\
\hline
\end{tabular}

rabbits [Bayar et al., 2001]. Primary side effects associated with topotecan and etoposide include myelosuppression, nausea, vomiting and alopecia.

\section{Ototoxicity Monitoring}

Audiological tests were conducted at St. Jude Children's Research Hospital. The monitoring protocol included otoscopy, tympanometry screening and measurements of TEOAEs. DPOAE measurements were also obtained in several of the children as described in Bhagat et al. [2010]. Due to time constraints, TEOAE and DPOAE measurements could not be completed in all children. All of the children completed a monitoring protocol during a baseline and an interim evaluation. The interim evaluation was conducted following the course of carboplatin at the midpoint of the child's chemotherapy regimen. This time point was chosen to determine if changes in TEOAE waveforms could be registered early in the course of chemotherapy. TEOAE tests in children $<12$ months of age at the onset of treatment were conducted in a suite. The older child was tested in a double-walled sound-treated booth. For 9 of the 10 children, the baseline evaluation took place prior to chemotherapy treatment. In the remaining child (No. 002), the baseline evaluation was conducted 1 week after the child had received one course of carboplatin. During the baseline and interim evaluations, 8 children were sedated during testing. Personnel from the Anesthesiology Department at St. Jude were present and actively monitored these children while they were sedated. One child was tested during natural sleep, and the remaining child (the oldest) was tested after completing pure-tone audiometry. Middle-ear function in the younger children was evaluated in both ears with a screening tympanometer (Maico, MI 24) capable of obtaining measurements with $226-$ or $1000-\mathrm{Hz}$ probe tones. The oldest child was tested with a diagnostic tympanometer (GSI TympStar) programmed for tympanometry screening. Tympanometry screening results suggested normal middle-ear function bilaterally in the children at the time of the baseline and interim evaluations. This was indicated by the presence of single-peaked 226- and/or $1000-\mathrm{Hz}$ tympanograms with a maximum admittance near 0 deca Pascals [Jerger, 1970; Alaerts et al., 2007]. Measurements of TEOAEs were obtained from both right and left ear canals in 8 of the children by means of an otoacoustic-emissions analyzer (Otodynamics, ILO 296) interfaced with a laptop computer (Dell Latitude D610). In the remaining 2 children (Nos. 010 and 014), comparisons between baseline and interim measurements were made in one ear due to the presence of cerumen impaction in the other ear. The ILO probe was calibrated using the $1-\mathrm{cm}^{3}$ cavity provided by the manufacturer prior to each baseline and interim test. The stimuli for TEOAE measurements consisted of $80-\mathrm{ms}$ clicks presented at a rate of 50 per second. The clicks were generated by the ILO v6 software program and transduced through the probe receiver at a targeted level of $70 \mathrm{~dB}$ peak equivalent (pe) SPL. A miniature microphone in the probe was used to detect the TEOAE response in the ear canal. The adequacy of the probe fit was checked prior to each TEOAE measurement by observing the stimulus spectrum in the ear canal and determining that the stimulus level closely matched the targeted level according to the guidelines recommended in the ILO v6 manual. The stimulus level in the ear canal is measured at a distance from the tympanic membrane. Data collection began after the fit of the probe was determined to be satisfactory. TEOAEs were averaged using the linear acquisition mode, which maximizes the signal-tonoise ratio of the TEOAE response in young children [Tognola et al., 2001]. TEOAE responses were collected for trains of 4 click stimuli of the same polarity and intensity. Two memory buffers, each of 20-ms duration, were used to store the TEOAE responses. The response for the first train of stimuli was stored in buffer $A$ and the response for the next train of stimuli was stored in buffer B. Each accepted sweep was the average of the responses to 8 stimuli (4 in buffer A and 4 in buffer B). Averaging of the TEOAE waveform was automatically terminated after 260 lownoise sweeps were collected.

\section{Wavelet Transform of TEOAEs}

As described previously, the TEOAE waveform exhibits 'frequency dispersion', meaning that the frequency content of the waveform varies along with time [Kemp, 1978]. The latency of appearance of frequency components in the TEOAE waveform is related to the frequency of the component: higher-frequency components have shorter latencies than lower-frequency components. The temporal organization of frequencies in the TEOAE waveform is consistent with place-frequency mapping in the cochlea, with basal regions tuned to high frequencies and apical regions tuned to lower frequencies. In this study, TEOAE waveforms were decomposed offline into a set of frequency components by WT analysis [Mallat, 1989]. The WT at the generic time $\tau$ and frequency $f$ of a signal $x(t)$ is defined as:

$$
W T(\tau, f)=\sqrt{f / f_{0}} \cdot \int x(t) \cdot w\left(f / f_{0}(t-\tau)\right) d t
$$

Basically, the WT decomposes a signal into elementary components by means of a bank of band-pass filters,

$$
\sqrt{f / f_{0}} \cdot w\left(f / f_{0}(t)\right),
$$

that are iteratively derived from a unique prototype function $w(t)$ called the mother wavelet. The mother wavelet $w(t)$ is a function with finite energy and centered around $t=0$; its Fourier transform is a band-pass function centered around frequency $f_{0}$. The bandwidth of the band-pass filters derived from the mother wavelet is proportional to the center frequency of the filter, but the duration of their impulse response in the time domain is inversely propor- 
tional to the center frequency of the filter (for a more detailed mathematical explanation, see Tognola et al. [1997, 1998]). The set of values WT $(\tau, f)$ calculated from equation (1) for each time $\tau$ and frequency $f$ is the time-frequency spectral distribution of the signal $x(t)$ and gives an indication as to which spectral components are present and at which times. An optimized mother wavelet has been developed to analyze TEOAEs [Tognola et al., 1998]:

$$
\mathrm{w}_{n}(t)=1 /\left(1+t^{2 n}\right) \cos (20 t),(n=2)
$$

By using equations (1) and (2), TEOAEs were decomposed into $100-\mathrm{Hz}$-wide (exact bandwidth $97.66 \mathrm{~Hz}$ ) frequency components in the approximate $500-5000 \mathrm{~Hz}$ range (exact frequency range $488-5078 \mathrm{~Hz}$ ) for a total of 48 frequency components for each TEOAE waveform. The main parameters evaluated for each waveform were the root-mean-square (rms) energy of the whole TEOAE signal and noise and the latency and the normalized energy for each of the 48 frequency components. The energy parameter is defined as the rms value in millipascals $(\mathrm{mPa})$ of the TEOAE signal and noise of each TEOAE. The signal was defined as $0.5^{*}(\mathrm{xA}+\mathrm{xB})$ and the noise was defined as $(\mathrm{xA}-\mathrm{xB}) / \sqrt{2}$, where $\mathrm{xA}$ and $\mathrm{xB}$ are the two replicate recordings (the $\mathrm{A}$ and $\mathrm{B}$ memory buffers). Similarly, the energy (rms value) of each of the 48 frequency components was computed using the same equation (2) by substituting $\mathrm{xA}$ and $\mathrm{xB}$ with the components extracted, at each frequency, from each of the two TEOAE recording buffers. The normalized energy is the percent value of the energy (rms value) of each frequency component normalized versus the energy of the broadband TEOAE. In this manner, if you sum up all of the normalized energy (\%) of each component, the total adds up to $100 \%$. The normalized energy percentage for each frequency component estimates the weight of each frequency component relative to the whole TEOAE energy. The latency of the 48 frequency components was extracted with WT analysis from each TEOAE and was defined as the time interval between the stimulus onset and the time sample where the envelope of the frequency component reached its maximum value.

\section{Data Analysis}

Data obtained from the right and left ears were statistically evaluated in separate analyses. For the parameters TEOAE latency and normalized energy, mean data were available for each of the 48 frequency components. In order to condense this information for statistical analysis purposes, data for both TEOAE latency and normalized energy were averaged across every six frequency components (corresponding to $600-\mathrm{Hz}$-wide bands) from approximately 500 to $5000 \mathrm{~Hz}$ for a total of 8 bands.

The data were statistically analyzed using the Statistical Package for the Social Sciences (SPSS) version 16.0 software. An alpha level of 0.05 was adopted for all statistical tests. Given the small sample size, Wilcoxon signed-rank tests were conducted on the whole TEOAE energy, and on the TEOAE latency and normalized energy in the 8 bands measured at the baseline and interim evaluations in order to determine if any significant differences were registered between these conditions. The Bonferroni correction was applied to adjust for type 1 error inflation for the statistical tests conducted on TEOAE latency and normalized energy in the 8 bands $(0.05 / 8=0.006)$. In addition to statistical analysis for the mean data, descriptive data on individual subjects were catalogued and individual trends were reported.

Time-Frequency Analysis of TEOAEs in Children on Carboplatin Chemotherapy
Table 2. TEOAE energy (in dB SPL) measured at the baseline and interim evaluations

\begin{tabular}{lccccc}
\hline \multirow{2}{*}{$\begin{array}{l}\text { Subject } \\
\text { No. }\end{array}$} & \multicolumn{2}{c}{ Baseline } & \multicolumn{3}{c}{ Interim } \\
\cline { 2 - 3 } \cline { 5 - 6 } \cline { 5 - 6 } & right ear & left ear & & right ear & left ear \\
\hline 002 & 23.14 & 20.08 & & 21.04 & 18.50 \\
003 & 11.62 & 11.17 & & 14.85 & 13.90 \\
004 & 20.42 & 14.29 & & 19.08 & 15.04 \\
005 & 7.91 & 9.40 & & 8.12 & 5.88 \\
006 & 19.53 & 20.77 & 18.90 & 14.27 \\
007 & 17.08 & 17.0 & & 13.01 & 12.07 \\
010 & - & 15.65 & & - & 21.62 \\
012 & 15.86 & 10.42 & & 17.25 & 13.0 \\
014 & 10.32 & - & 9.39 & - \\
016 & 22.46 & 18.91 & 30.83 & 26.02 \\
\hline
\end{tabular}

\section{Results}

The whole TEOAE energy at the baseline and interim measurements is shown for each subject in table 2. For purposes of comparison with other studies, these whole TEOAE energy values were converted from $\mathrm{mPa}$ into decibels re: 20 micropascals (dB SPL). Subject No. 002 received one course of chemotherapy before his baseline measurement could be completed; as can be seen, this baseline measurement was not clearly below those of the other children in the study, suggesting that the one course of chemotherapy did not adversely bias baseline measurements in this child. Examples of TEOAE waveforms for individual subjects can be seen in figures 1 and 2. As can be seen, there are changes in the amplitude of TEOAE waveforms, but no common trend could be observed for all of the ears. There were some ears in which the amplitude of the TEOAE decreased at the interim measurement compared to the baseline measurement, and others where it increased at the interim measurement compared to the baseline measurement. The TEOAE energy over the whole group of ears appeared not to change between conditions. The results of the statistical analysis indicated that there was no statistically significant difference in baseline and interim TEOAE signal energy or noise energy between conditions ( $\mathrm{p}>0.05$ ).

TEOAE latency is plotted for each ear across the 8 bands derived from the 48 frequency components in figure 3. These curves follow the well-known trends of higher frequencies having shorter latencies and lower frequencies having longer latencies. In normal hearing subjects, this trend between latency and frequency is well 
Fig. 1. TEOAEs measured at baseline (a) and at interim evaluation (b) from the right ears of 5 children. Numbers on the right are the child IDs.
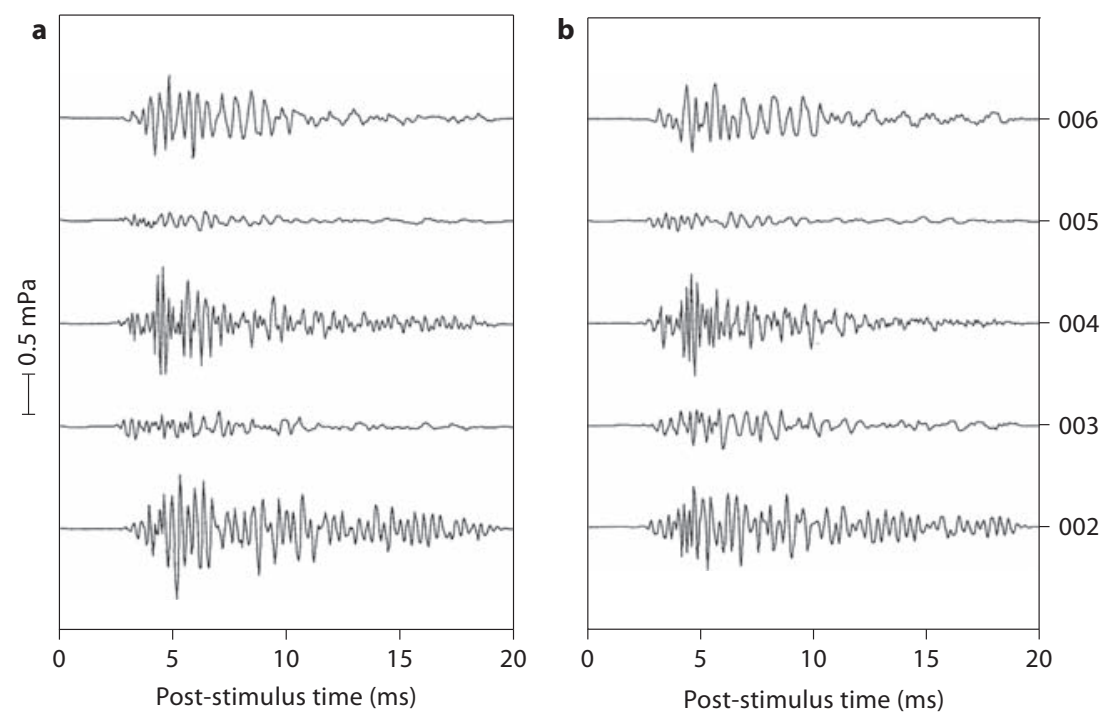
and at interim evaluation (b) from the left ears of 5 children. Numbers on the right are the child IDs.
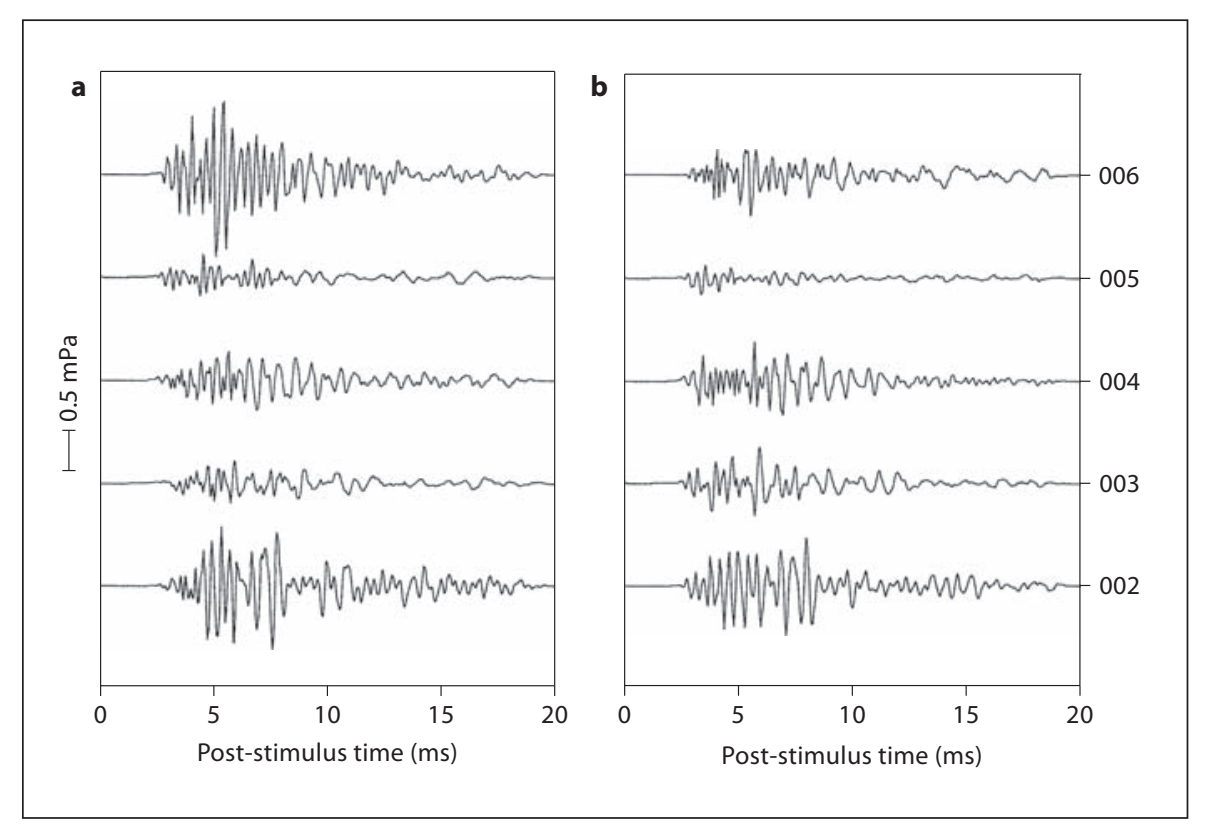

described by a power law model, as shown by Tognola et al. [1997]:

$$
L A T=a \cdot f^{b}
$$

where LAT and $f$ are the latency (ms) and the frequency (in $\mathrm{kHz}$ ) of the TEOAE component, and $a$ and $b$ are constant parameters. Normative data derived from adults with well-functioning OHCs [Tognola et al., 1997], showed that the parameter $b$ is almost not influenced by the intensity of the click stimuli and ranges from -0.44 to -0.40 , whereas the parameter $a$ depends on the intensity of the click stimuli. As shown in figure 3, the latency curves computed before and during carboplatin treatment were very similar and did not reveal systematic differences between the baseline and interim measurements. This was the case for both right and left ears. The statisti- 

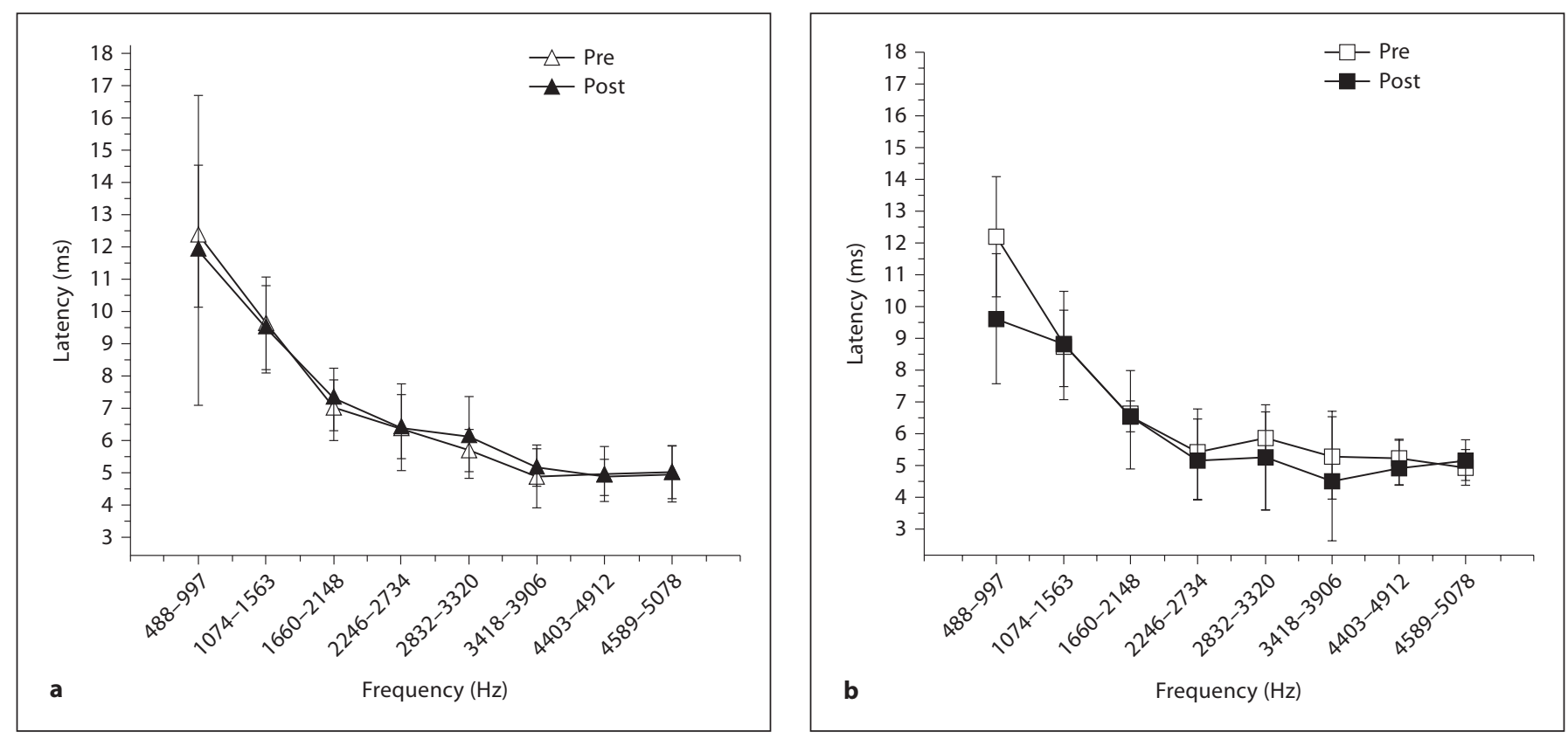

Fig. 3. a Median (interquartile range) TEOAE latency in the 8 frequency bands spanning the $488-5078 \mathrm{~Hz}$ range derived from the $48 \mathrm{WT}$ frequency components. Results from right ears are depicted with triangles. b Median (interquartile range) TEOAE latency in the 8 frequency bands spanning the $488-5078 \mathrm{~Hz}$ range derived from the 48 WT frequency components. Results from left ears are depicted with squares.
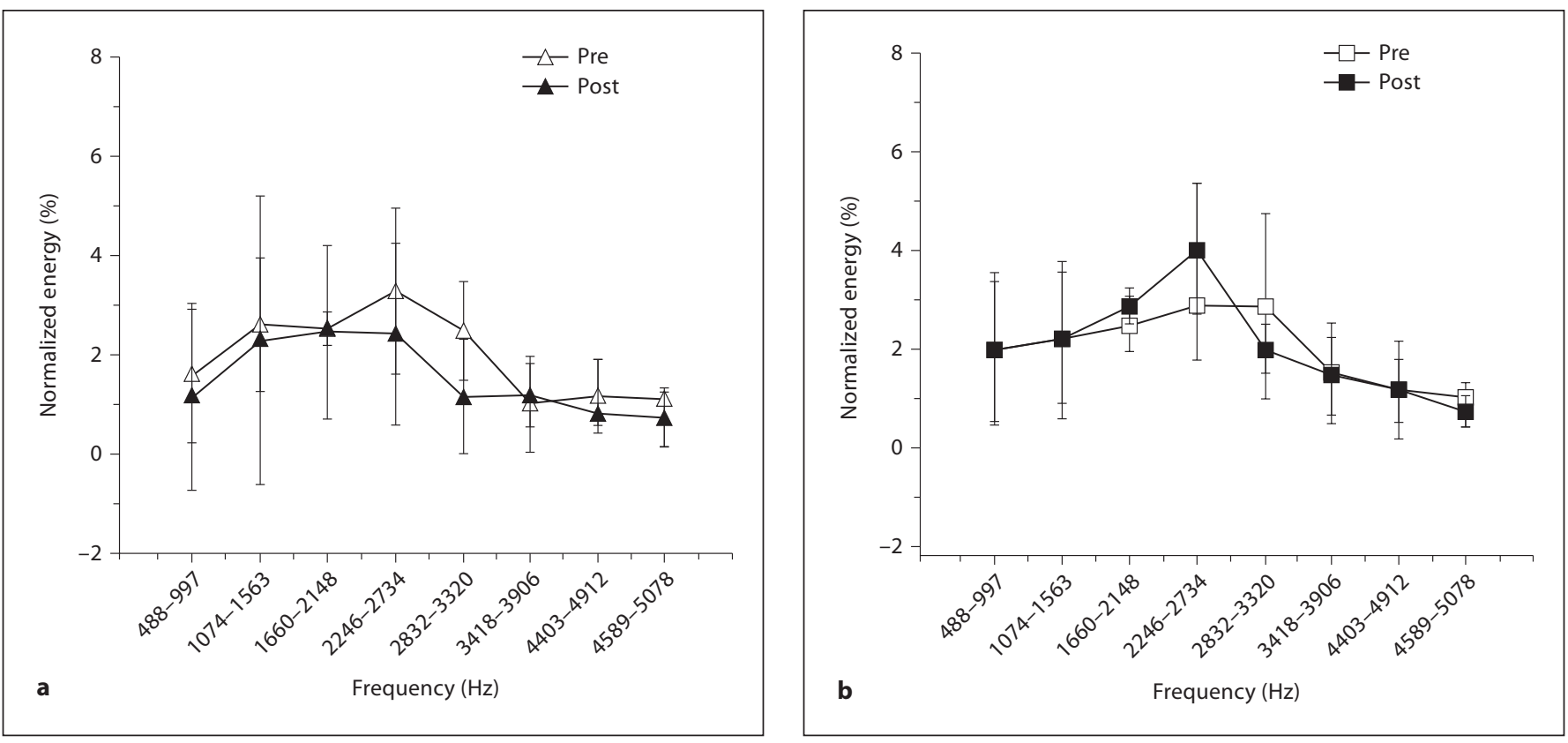

Fig. 4. a Median (interquartile range) normalized energy of TEOAEs in the 8 frequency bands spanning the 488-5078 Hz range derived from the 48 WT frequency components. Results from right ears are depicted with triangles. b Median (interquartile range) normalized energy of TEOAEs in the 8 frequency bands spanning the $488-5078 \mathrm{~Hz}$ range derived from the $48 \mathrm{WT}$ frequency components. Results from left ears are depicted with squares. 
cal analysis revealed no significant differences $(p>0.05)$ in latency between conditions for either right or left ears. TEOAE normalized energy for the 8 bands derived from the 48 frequency components is shown in figure 4 . Although there were nominal decreases in the values of normalized energy particularly in the 2246-2734 and 2832$3320 \mathrm{~Hz}$ bands in both ears, no statistically significant differences in these frequency bands were observed. Tables 3-5 display the statistical test results for the analyses performed on the whole TEOAE energy and on the TEOAE latency and normalized energy in the 8 bands.

Different from what was observed in the pooled data, decreases in the $2000-3000 \mathrm{~Hz}$ range were found in some individual subject data. This was observed in TEOAEs recorded in 10/18 ears that were included in the sam- ple studied. As examples, figure 5 depicts changes in TEOAE normalized energy in 4 individual ears when comparing the baseline measurement to the interim measurement.

\section{Discussion}

The aims of this study were to characterize and quantify time-frequency changes in TEOAEs recorded in children diagnosed with retinoblastoma who were receiving carboplatin chemotherapy. WT analysis of TEOAEs provides a novel means to examine potential ototoxicity in the pediatric population with a methodology that offers several advantages over conventional FFT analysis of

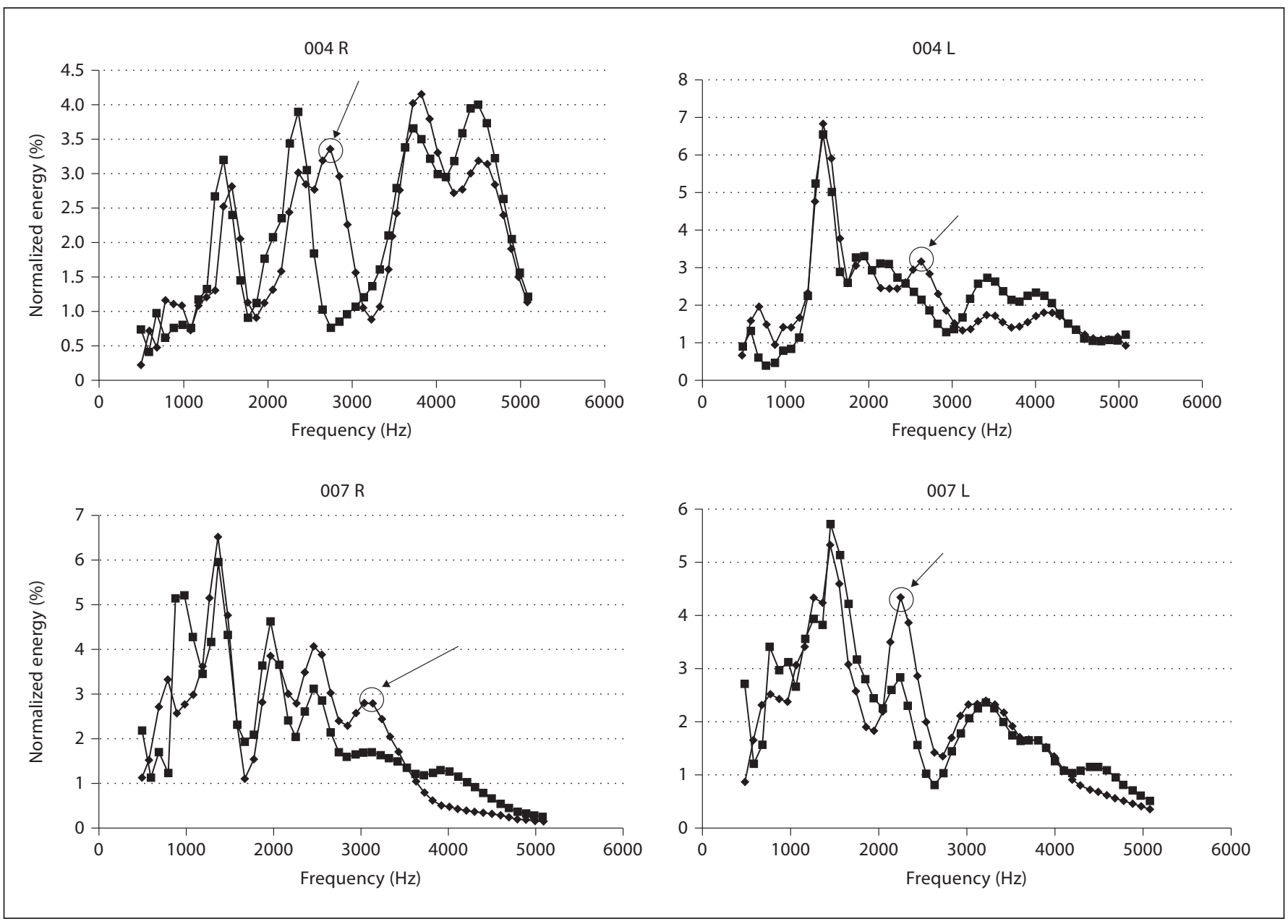

Fig. 5. Normalized energy of the 48 WT frequency components of TEOAEs measured at baseline (diamonds) and at interim evaluation (squares) of subject No. 004 and subject No. 007. Arrows indicate frequency regions where reductions in TEOAE normalized energy were prominent. $\mathrm{L}=\mathrm{Left}$; $\mathrm{R}=$ right. 
TEOAE waveforms. In infants and young children, behavioral hearing tests can often be difficult to conduct and difficult to interpret. The methods used to evaluate hearing behaviorally in the youngest children often lack frequency specificity. Measurement of TEOAEs offers

Table 3. Statistical test results using the Wilcoxon signed-rank test to compare baseline (pre-carboplatin treatment) and interim (post-carboplatin treatment) whole TEOAE energy

\begin{tabular}{lcc}
\hline & Right ears & Left ears \\
\hline$Z$ & $-0.06^{\mathrm{a}}$ & $-0.18^{\mathrm{b}}$ \\
p value & 0.95 & 0.86 \\
\hline
\end{tabular}

The $\mathrm{p}$ value is displayed for each comparison. An alpha level of 0.05 was used for all statistical tests.

${ }^{a}$ Based on positive ranks; ${ }^{b}$ based on negative ranks. a useful alternative to behavioral audiometry, in that TEOAEs do not require children to actively participate in the test and can offer information on cochlear integrity in specific frequency regions. In addition, the TEOAE waveform can be subjected to powerful signal processing techniques, such as WT analysis, which can adequately quantify changes in time-varying signals. As described earlier in the text (see Introduction), the WT analysis has the potential to accurately assess possible subtle auditory dysfunctions that may signal the onset of ototoxicity.

The results of this study indicated that on a group basis, no statistically significant differences in WT measurements of TEOAE energy, latency or normalized energy occurred when comparing TEOAE measurements obtained before and after 3-4 courses of carboplatin chemotherapy. These results are consistent with previous studies of OAEs in children with retinoblastoma receiving carboplatin chemotherapy. Bhagat et al. [2010] found

Table 4. Statistical test results using the Wilcoxon signed-rank test to compare baseline (pre-carboplatin treatment) and interim (postcarboplatin treatment) TEOAE latency in 8 frequency bands

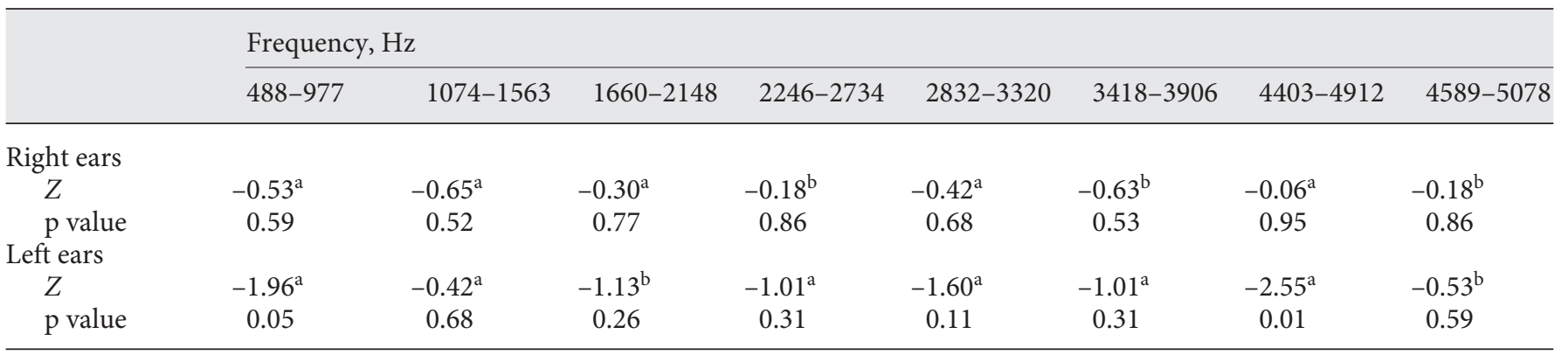

The $\mathrm{p}$ value is displayed for each comparison. An alpha level (Bonferroni-corrected) of 0.05/8 $=0.006$ was used for all statistical tests. ${ }^{a}$ Based on positive ranks; ${ }^{b}$ based on negative ranks.

Table 5. Statistical test results using the Wilcoxon signed-rank test to compare baseline (pre-carboplatin treatment) and interim (postcarboplatin treatment) normalized energy in 8 frequency bands

\begin{tabular}{|c|c|c|c|c|c|c|c|c|}
\hline & \multicolumn{8}{|c|}{ Frequency, $\mathrm{Hz}$} \\
\hline$Z$ & $-0.77^{\mathrm{a}}$ & $-0.53^{\mathrm{b}}$ & $-0.42^{\mathrm{a}}$ & $-1.13^{\mathrm{a}}$ & $-1.96^{\mathrm{a}}$ & $-0.30^{\mathrm{a}}$ & $-0.89^{\mathrm{a}}$ & $-1.72^{\mathrm{a}}$ \\
\hline $\mathrm{p}$ value & 0.44 & 0.59 & 0.68 & 0.26 & 0.05 & 0.77 & 0.37 & 0.09 \\
\hline \multicolumn{9}{|l|}{ Left ears } \\
\hline
\end{tabular}

The $\mathrm{p}$ value is displayed for each comparison. An alpha level (Bonferroni-corrected) of 0.05/8 $=0.006$ was used for all statistical tests. ${ }^{a}$ Based on positive ranks; ${ }^{b}$ based on negative ranks. 
that the mean DPOAE levels were reduced after carboplatin chemotherapy in these children only at the highest frequency tested, which was $7996 \mathrm{~Hz}$. The highest TEOAE frequency evaluated in this study was approximately $5000 \mathrm{~Hz}$, and so the results seen in this investigation agree with the findings of the previous study. Similarly, Smits et al. [2006] found little evidence of a carboplatin-induced change in OAEs in their sample of children with retinoblastoma receiving chemotherapy. In older children, the literature indicates that the incidence of carboplatin-induced hearing loss after chemotherapy as measured on the audiogram in youngsters afflicted with retinoblastoma is variable, with as few as $6.6 \%$ of children [Pecora Liberman et al., 2011] to as many as $15.3 \%$ of children [Namouni et al., 1997]. While carboplatin ototoxicity is uncommon immediately following the completion of the chemotherapy regimen, long-term studies of children receiving carboplatin for retinoblastoma indicate that cases of delayed hearing loss can occur years after children complete the chemotherapy regimen [Jehanne et al., 2009]. In a retrospective review of 60 patients with retinoblastoma treated with carboplatin at St. Jude Children's Research Hospital, Qaddoumi et al. [2012] found that 10/60 patients (17\%) had developed permanent hearing loss sometime after the initiation of treatment. Four patients were identified as having hearing loss years after the initiation of treatment. Importantly, children less than 6 months of age at the onset of treatment were at significantly greater risk of developing carboplatin-induced hearing loss compared to children older than this.

Identification of which children are more at risk of developing carboplatin ototoxicity later in life is a high priority. When carboplatin induces hearing loss, it is usually initially manifested first at frequencies higher than $4000 \mathrm{~Hz}$ [Macdonald et al., 1994]. It is conceivable that initial changes in $\mathrm{OHC}$ function would be localized initially to higher frequencies. Further exploration of this hypothesis would be aided by examining TEOAEs acquired with acoustic stimulation higher than $5000 \mathrm{~Hz}$. Although there is a research effort aimed at evaluating high-frequency TEOAE tests, most of these studies have been conducted in adults [Goodman et al., 2009; Keefe et al., 2011]. Evaluation of high-frequency TEOAE tests in normal-hearing infants and young children and the creation of a normative database would facilitate the clinical application of this method, particularly in pediatric populations exposed to ototoxic agents.

When monitoring for platinum-compound ototoxicity, clinicians often compare results from pretherapy tests to posttherapy tests in individual subjects. Therefore, noting changes in TEOAE waveforms on an individual basis can be informative. In our investigation, there were decreases in normalized energy on an individual basis in the $2000-3000 \mathrm{~Hz}$ region in $10 / 18$ ears studied in the sample. This result indicates that the frequency content of the TEOAE waveform had changed following exposure to carboplatin. Some of the frequency components within this frequency range which were predominant before the exposure lost energy following treatment with carboplatin. It remains to be seen if these changes indicate a predisposition for the development of hearing loss, since most of the children examined in this study did not receive behavioral hearing threshold tests due to their young age. However, it is curious that a decrease in normalized energy in the $2000-3000 \mathrm{~Hz}$ was a common finding amongst several of the children. Bhagat et al. [2010] found that $4 / 10$ children in this protocol had criterion reductions in DPOAE levels following carboplatin chemotherapy. Two of these children (Nos. 002 and 004) exhibited DPOAE level reductions in the 2000$4000 \mathrm{~Hz}$ frequency range. Studies in chinchillas have shown that round window exposure to a high dose of carboplatin $(16 \mathrm{mg} / \mathrm{ml})$ could cause a progressive loss of OHCs beginning in the $1000 \mathrm{~Hz}$ region and increasing through the basal turn of the cochlea [Bauer and Brozowski, 2005]. The need to provide children receiving carboplatin with long-term monitoring is apparent given the potential development of delayed-onset hearing loss.

The use of WT analysis of TEOAE waveforms is a powerful methodology that has yet to be applied to monitoring for ototoxicity in the pediatric cancer population. It has been proven that WT analysis can provide useful information that can supplement the information provided by other techniques used to evaluate TEOAE waveforms, including FFT analysis. This additional information includes the ability to quantify TEOAE energy and latency in multiple frequency bands that conventional measurement techniques typically cannot provide. The analysis of latency of TEOAE components is of great help to improve the detection of subtle and subclinical changes in cochlear mechanisms, thanks to the high sensitivity of latency to possible subtle OHC dysfunction [Moleti et al., 2005] or to possible changes in the mechanical properties of the basilar membrane [Sisto and Moleti, 2002]. As the latency of TEOAE components gives an estimate of the cochlear round-trip delay from the base of the cochlea to the tonotopic place and back, possible alterations in latency of TEOAE components could suggest 
alterations in the mechanical properties of the basilar membrane, cochlear tuning and propagation velocity of the traveling wave [Andoh and Wada, 2004; Moleti et al., 2005]. Also, as argued by Tognola et al. [1999] and Jedrzejczak et al. [2005], alterations in latency of TEOAE components could also be an indicator of local functional impairment of OHCs, that would result in a shift of the cochlear response in adjacent (well-functioning) regions and thus in changes in the length of the cochlear traveling path.

Although it is not yet commercially available, the use of WT analysis of TEOAE waveforms is a novel methodology that should be applied to monitoring for ototoxicity in the pediatric cancer population. Future research, including finding evidence of the early onset of ototoxicity caused by other platinum compounds (e.g. cisplatin), would benefit from the application of WT analysis of TEOAEs.

\section{Conclusions}

Comparison between group baseline and interim evaluations revealed no significant decreases in WT analysis of TEOAEs for the parameters energy, latency and normalized energy after 3-4 courses of carboplatin chemotherapy. On an individual basis, 10/18 ears in the sample exhibited decreases in normalized energy in the 2000 $3000 \mathrm{~Hz}$ range following exposure to carboplatin. Monitoring of ototoxicity in children receiving platinum compounds should include WT analysis of TEOAE waveforms.

\section{Acknowledgments}

This work was supported in part by grants CA 21765 and CA 23099 from the US Public Health Service and by the American Lebanese Syrian Associated Charities. We wish to thank Tammy Free and Vicki Given for their contributions to this project.

\section{References}

Abramson DH, Frank CM, Susman M, Whalen MP, Dunkel IJ, Boyd NW 3rd: Presenting signs of retinoblastoma. J Pediatr 1998;132: 505-508.

Alaerts J, Luts H, Wouters J: Evaluation of middle ear function in young children: clinical guidelines for the use of 226- and $1,000-\mathrm{Hz}$ tympanometry. Otol Neurotol 2007;28:727732.

Allen GC, Tiu C, Koike K, Ritchey AK, KursLasky M, Wax MK: Transient-evoked otoacoustic emissions in children after cisplatin chemotherapy. Otolaryngol Head Neck Surg 1998; 118:584-588.

Andoh M, Wada H: Prediction of the characteristics of two types of pressure waves in the cochlea: theoretical considerations. J Acoust Soc Am 2004; 116:417-425.

- Bacha DM, Caparros-Sison B, Allen JA, Walker R, Tan CT: Phase I study of carboplatin (CBDCA) in children with cancer. Cancer Treat Rep 1986;70:865-869.

- Bauer CA, Brozowski TJ: Cochlear structure and function after round window application of ototoxins. Hear Res 2005;201:121-131.

- Bayar N, Boke B, Apan A, Koc MC: Efficacy of topotecan treatment on an experimental model of transient evoked otoacoustic emissions. Int J Pediatr Otorhinlarngol 2001;61: 135-142.

Bertolini P, Lassalle M, Mercier G, Raquin MA, Izzi G, Corradini N, Hartmann O: Platinum compound-related ototoxicity in children: long term follow-up reveals continuous worsening of hearing loss. J Pediatr Hematol Oncol 2004;26:649-655.

Time-Frequency Analysis of TEOAEs in Children on Carboplatin Chemotherapy
Bhagat SP, Bass JK, White ST, Qaddoumi I, Wilson MW, Wu J, Rodriguez-Galindo C: Monitoring carboplatin ototoxicity with distortion-product otoacoustic emissions in children with retinoblastoma. Int $J$ Pediatr Otorhinolaryngol 2010;74:1156-1163.

- Broaddus E, Topham A, Singh AD: Incidence of retinoblastoma in the USA: 1975-2004. Br J Opthalmol 2009;93:21-23.

Brownell WE: Outer hair cell electromotility and otoacoustic emissions. Ear Hear 1990;11:8292.

Dean JB, Hayashi SS, Albert CM, King AA, Karson R, Hayashi RJ: Hearing loss in pediatric oncology patients receiving carboplatincontaining regimens. J Pediatr Hematol Oncol 2008;30:130-134.

Goodman SS, Fitzpatrick DF, Ellison JC, Jesteadt W, Keefe DE: High frequency click-evoked otoacoustic emissions and behavioral thresholds in humans. J Acoust Soc Am 2009;125:1014-1032.

Hofstetter P, Ding D, Powers N, Salvi R: Quantitative relationship of carboplatin dose to magnitude of inner and outer hair call loss and the reduction of distortion product otoacoustic emission amplitude in chinchillas. Hear Res 1997;112:199-215.

- Jedrzejczak WW, Blinowska KJ, Konopka W: Time-frequency analysis of transiently evoked otoacoustic emissions of subjects exposed to noise. Hear Res 2005;205:249-255.
Jehanne M, Lumbroso-Le Rouic L, Savignoni A, Aerts I, Mercer G, Bours D, Desjardins L, Doz F: Analysis of ototoxicity in young children receiving carboplatin in the context of conservative management of unilateral or bilateral retinoblastoma. Pediatr Blood Cancer 2009;52:637-643.

Jerger J: Clinical experience with impedance audiometry. Arch Otolaryngol 1970;92:311324

Keefe DH, Goodman SS, Ellison JC, Fitzpatrick DF, Gorga MP: Detecting high-frequency hearing loss with click-evoked otoacoustic emissions. J Acoust Soc Am 2011;129:245261.

Kemp DT: Stimulated acoustic emissions from within the human auditory system. J Acoust Soc Am 1978;64:1386-1391.

-Knight KR, Kraemer DF, Neuwelt EA: Ototoxicity in children receiving platinum chemotherapy: underestimating a commonly occurring toxicity that may influence academic and social development. J Clin Oncol 2005; 23:8588-8596.

Knight KR, Kraemer DF, Winter C, Neuwelt EA: Early changes in auditory function as a result of platinum chemotherapy: use of extended high-frequency audiometry and evoked distortion product otoacoustic emissions. J Clin Oncol 2007:25:1190-1195.

Li Y, Womer RB, Silber JH: Predicting cisplatin ototoxicity in children: the influence of age and the cumulative dose. Eur J Cancer 2004; 40:2445-2551. 
- Macdonald MR, Harrison RV, Wake M, Bliss B, Macdonald RE: Ototoxicity of carboplatin: comparing animal and clinical models at the Hospital for Sick Children. J Otolaryn 1994; 23:151-159.

Mallat SG: A theory for multiresolution signal decomposition: the wavelet representation. IEEE Pattern Anal Mach Intell 1989;11:674-693.

- Marozas V, Janusauskas A, Lukosevicius A, Sörnmo L: Multiscale detection of transient evoked otoacoustic emissions. IEEE Trans Biomed Eng 2006;53:1586-1593.

Moleti A, Sisto R, Tognola G, Parazzini M, Ravazzani $P$, Grandori F: Otoacoustic emission latency, cochlear tuning, and hearing functionality in neonates. J Acoust Soc Am 2005; 118:1576-1584.

Namouni F, Doz F, Tanquy ML, Quintana E, Michon J, Pacquement H, Bouffet E, Gentet JC, Plantaz D, Lutz P, Vannier JP, Validire P, Neuenschwander S, Desjardins L, Zucker JM: High-dose chemotherapy with carboplatin, etoposide and cyclophosphamide followed by a haematopoietic stem cell rescue in patients with high-risk retinoblastoma: a SFOP and SFGM study. Eur J Cancer 1997;33:2368-2375.

Paglialonga A, Barozzi S, Brambilla D, Soi D, Cesarani A, Gagliardi C, Comiotto E, Spreafico E, Tognola G: Cochlear active mechanisms in young normal-hearing subjects affected by Williams syndrome: time-frequency analysis of otoacoustic emissions. Hear Res 2011a; 272:157-167.

- Paglialonga A, Fiocchi S, Del Bo L, Ravazzani P, Tognola G: Quantitative analysis of cochlear active mechanisms in tinnitus subjects with normal hearing sensitivity: time-frequency analysis of transient evoked otoacoustic emissions and contralateral suppression. Auris Nasus Larynx 2011b;38:33-40.

-Paglialonga A, Tognola G, Parazzini M, Lutman ME, Bell SL, Thuroczy G, Ravazzani P: Effects of mobile phone exposure on time frequency fine structure of transiently evoked otoacoustic emissions. J Acoust Soc Am 2007;122:2174-2182.
Parsons SK, Neault MW, Lehmann LE, Brennan LL, Eickhoff CE, Kretschmar CS, Diller LR: Severe ototoxicity following carboplatin-containing conditioning regimen for autologous marrow transplantation for neuroblastoma. Bone Marrow Transplant 1998;22:669-674.

Pecora Liberman PH, Schultz C, Schmidt GoffiGómez MV, Antoneli CB, Motoro Chojniak M, Eduardo Novaes P: Evaluation of ototoxicity in children treated for retinoblastoma: preliminary results of a systematic audiological evaluation. Clin Transl Oncol 2011;13: 348-352.

Qaddoumi I, Bass JK, Wu J, Billups CA, Wozniak AW, Merchant TE, Haik BG, Wilson MW, Rodriguez-Galindo C: Carboplatin-associated ototoxicity in children with retinoblastoma. J Clin Oncol 2012;30:1034-1041.

Riga M, Psarommatis I, Korres S, Lyra C, Papadeas E, Varvutsi M, Ferekidis E, Apostolopouos N: The effect of treatment with vincristine on transient evoked and distortion product otoacoustic emissions. Int J Pediatr Otorhinolaryngol 2006;70:1003-1008.

Rodriguez-Galindo C, Wilson MW, Haik BG, Merchant TE, Billups CA, Shah N, Cain A, Langston J, Lipson M, Kun LE, Pratt CB: Treatment of intraocular retinoblastoma with vincristine and carboplatin. J Clin Oncol 2003;21:2019-2025.

Saito T, Saito H, Saito K, Wakui S, Manabe Y, Tsuda G: Ototoxicity of carboplatin in guinea pigs. Auris Nasus Larynx 1989;16:13-21.

- Simon T, Hero B, Dupuis W, Selle B, Berthold F: The incidence of hearing impairment after successful treatment of neuroblastoma. Klin Padiatr 2002;214:149-152.

Sisto R, Moleti A: On the frequency dependence of the otoacoustic emission latency in hypoacoustic and normal ears. J Acoust Soc Am 2002;111:297-308.

Smits C, Swen SJ, Theo Goverts ST, Moll AC, Imhof SM, Schouten-van Meeteren AY: Assessment of hearing in very young children receiving carboplatin for retinoblastoma. Eur J Cancer 2006;42:492-500.

-Stavroulaki P, Apostolopoulos N, Segas J, Tsakanikos M, Adamopoulos G: Evoked otoacoustic emissions - an approach for monitoring cisplatin induced ototoxicity in children. Int J Pediatr Otorhinolaryngol 2001; 59:47-57.
Stern JW, Bunin N: Prospective study of carboplatin-based chemotherapy for pediatric germ cell tumors. Med Pediatr Oncol 2002; 39:163-167.

- Takeno S, Harrison RV, Ibrahim D, Wake M, Mount RJ: Cochlear function after selective inner hair cell degeneration induced by carboplatin. Hear Res 1994;75:93-102.

Tognola G, Grandori F, Ravazzani P: Time-frequency distributions of click-evoked otoacoustic emissions. Hear Res 1997; 106:112122.

Tognola G, Grandori F, Ravazzani P: Wavelet analysis of click-evoked otoacoustic emissions. IEEE Trans Biomed Eng 1998;45:686697.

Tognola G, Grandori F, Avan P, Ravazzani P, Bonfils P: Frequency-specific information from click evoked otoacoustic emissions in noise-induced hearing loss. Audiology 1999; 38:243-250.

- Tognola G, Ravazanni P, Molini E, Ricci G, Alunni N, Parazanni M, Grandori F: 'Linear' and 'derived' otoacoustic emissions in newborns: a comparative study. Ear Hear 2001; 22:182-190.

- Yang LP, Young ST, Ku TS: Modification of the wavelet method used in transiently evoked otoacoustic emission pass/fail criterion to increase its accuracy. Med Biol Eng Comput 2002;40:34-40.

Yardley MP, Davies, CM, Stevens, JC: Use of transient evoked otoacoustic emissions to detect and monitor cochlear damage caused by platinum-containing drugs. Br J Audiol 1998;32:305-316.

-Yilmaz S, Oktem F, Karaman E: Detection of cisplatin-induced ototoxicity with transient evoked otoacoustic emission test before pure tone audiometer. Eur Arch Otorhinolaryngol 2010;267 1041-1044.

Zhang ZG, Zhang VW, Chan SC, McPherson B, $\mathrm{Hu}$ Y: Time-frequency analysis of clickevoked otoacoustic emissions by means of a minimum variance spectral estimationbased method. Hear Res 2008;243:18-27. 